\title{
On Generalized Dual Euler Angles
}

\author{
Aleix Rull and Federico Thomas \\ Institut de Robòtica i Informàtica Industrial (CSIC-UPC), Spain \\ e-mail: \{arull, fthomas\}@iri.upc.edu
}

\begin{abstract}
This paper first explores the generalization of Euler angles to the case in which the rotation axes are not necessarily members of an orthonormal triad, and presents a concise solution to their computation that relies on the calculation of standard Euler angles. Then, this generalization is taken one step further by introducing translations, that is, by defining generalized Euler angles about screw axes using a variation of the principle of transference that avoids the use of dual numbers. As an example, the obtained formulation is applied to solve the inverse kinematics of a $3 \mathrm{C}$ manipulator.
\end{abstract}

Key words: Euler angles, generalized Euler angles, dual Euler angles, 3C manipulator.

\section{Introduction}

Decomposing a rotation into three partial rotations about prescribed axes is considered an important problem in the parametrization of the threedimensional rotation group [1] with applications to motion planning and inverse kinematics. For example, the inverse kinematics of a wrist-partitioned manipulator requires the decomposition of a given finite rotation of the endeffector into three successive finite rotations about prescribed joint axes. When these three axes are selected from an orthogonal basis, the rotation angles are the well-known Euler angles. The case in which the three axes are not necessarily orthogonal was first treated by Davenport in 1973 [2]. He considered the case in which the first and the third axes were orthogonal to the second, but the angle between the first and third was arbitrary. In 1978, Dimentberg took the problem in all its generality and reduced it to three quadratic equations each relating the tangent of the half input angle with the tangents of the half rotations angles [3]. In 1992, Wohlhart obtained a similar expression for the first rotation angle and two linear equations relating the tangents of the first angle and each of the other two angles, thus improving Dimentberg's formulation [4]. The main problem of these two formulations is their discontinuity at $\pm \pi$. In 2003, Wittenburg and Lilov [5], and Shus- 
ter and Markley [6], independently gave a successful solution to the problem that avoids this formulation singularity. More recently, in 2011, Mladenova and Mladenov solved the problem using a vector-like parametrization of the rotation group [7], and in 2012 Piovan a Bullo presented a solution based on a coordinate-free formulation [8].

Another generalization of Euler angles consists in introducing translations, that is, in defining Euler angles about screw axes [9]. This leads to the concept of dual Euler angles which has successfully been used in several biomechanical applications $[10,11]$. A dual angle, say $\hat{\phi}$, is defined as a dual number of the form $\phi+\epsilon d$, with $\epsilon^{2}=0$, so that $\phi$ is the rotation about and $d$ the slide along a given axis. Dual numbers were introduced in the 19th century by Clifford, and their application to rigid body kinematics was subsequently generalized by Kotelnikov and Study in their principle of transference. This principle essentially states that, if dual angles replace real ones, then all equations obtained for spherical problems are also valid for spatial ones [12]. Therefore, general coordinate transformations can be expressed as a sequence of rotations through three dual Euler angles.

This papers deals with the problem of computing dual Euler angles for the case in which the three rotation axes are not mutually orthogonal, thus giving a unified treatment of the two generalizations of Euler angles available in the kinematics literature. It is interesting to observe that this problem was implicitly solved by Pennock and Vierstra in 1990 when calculating the inverse kinematics of the general $3 \mathrm{C}$ manipulator [15]. Curiously enough, this connection remained unnoticed in all subsequent works dealing with generalizations of Euler angles. One of the purposes of this paper is to highlight this connection and to provide a more concise and readable formulation to that given in [15].

The rest of this paper is organized as follows. In Section 2, by relying on the computation of standard Euler angles, a concise way to compute generalized Euler angles is presented. Next, in Section 3, this result is extended to dual angles using an alternative formulation of the principle of transference that makes no explicit use of dual numbers. The derived equations are then used, in Section 4, to solve the inverse kinematics of a 3C manipulator. Finally, conclusions are drawn in Section 5.

\section{Computing sets of generalized Euler angles}

The problem of decomposing a rotation about an axis $\mathbf{n}$ by an angle $\phi$ into three successive rotations about three axes $\mathbf{n}_{1}, \mathbf{n}_{2}$, and $\mathbf{n}_{3}$ by angles $\alpha_{1}, \alpha_{2}$, and $\alpha_{3}$, respectively, can be algebraically expressed as:

$$
\mathbf{R}_{\mathbf{n}}(\phi)=\mathbf{R}_{\mathbf{n}_{1}}\left(\alpha_{1}\right) \mathbf{R}_{\mathbf{n}_{2}}\left(\alpha_{2}\right) \mathbf{R}_{\mathbf{n}_{3}}\left(\alpha_{3}\right) .
$$




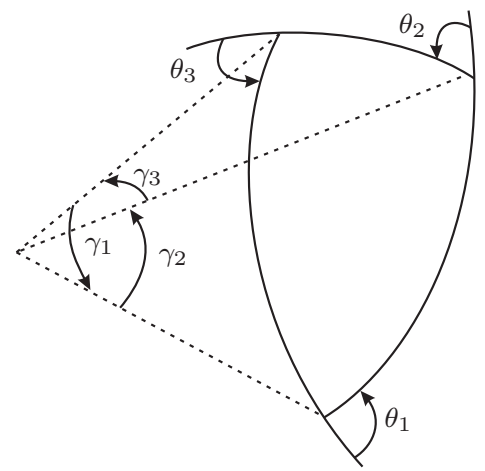

Fig. 1 Spherical triangle associated with matrix equation (2).

Expressing the rotations about $\mathbf{n}_{1}, \mathbf{n}_{2}$, and $\mathbf{n}_{3}$ in terms of rotations about the $\mathbf{z}$ axis, we have:

$$
\mathbf{C}_{1} \mathbf{R}_{\mathbf{z}}\left(\alpha_{1}\right) \mathbf{C}_{1}^{T} \mathbf{C}_{2} \mathbf{R}_{\mathbf{z}}\left(\alpha_{2}\right) \mathbf{C}_{2}^{T} \mathbf{C}_{3} \mathbf{R}_{\mathbf{z}}\left(\alpha_{3}\right) \mathbf{C}_{3}^{T}=\mathbf{R}_{\mathbf{n}}(\phi) .
$$

Rearranging terms, this can be rewritten as:

$$
\mathbf{C}_{3}^{T} \mathbf{R}_{\mathbf{n}}^{T}(\phi) \mathbf{C}_{1} \mathbf{R}_{\mathbf{z}}\left(\alpha_{1}\right) \mathbf{C}_{1}^{T} \mathbf{C}_{2} \mathbf{R}_{\mathbf{z}}\left(\alpha_{2}\right) \mathbf{C}_{2}^{T} \mathbf{C}_{3} \mathbf{R}_{\mathbf{z}}\left(\alpha_{3}\right)=\mathbf{I} .
$$

Then, computing the products of all constant matrices, we obtain:

$$
\mathbf{A}_{1} \mathbf{R}_{\mathbf{z}}\left(\alpha_{1}\right) \mathbf{A}_{2} \mathbf{R}_{\mathbf{z}}\left(\alpha_{2}\right) \mathbf{A}_{3} \mathbf{R}_{\mathbf{z}}\left(\alpha_{3}\right)=\mathbf{I} .
$$

Now, after computing a set of valid ZXZ Euler angles for $\mathbf{A}_{i}, i=1,2,3$, we have:

$$
\begin{aligned}
& \mathbf{R}_{\mathbf{z}}\left(\beta_{1}\right) \mathbf{R}_{\mathbf{x}}\left(\gamma_{1}\right) \mathbf{R}_{\mathbf{z}}\left(\delta_{1}\right) \mathbf{R}_{\mathbf{z}}\left(\alpha_{1}\right) \mathbf{R}_{\mathbf{z}}\left(\beta_{2}\right) \mathbf{R}_{\mathbf{x}}\left(\gamma_{2}\right) \mathbf{R}_{\mathbf{z}}\left(\delta_{2}\right) \mathbf{R}_{\mathbf{z}}\left(\alpha_{2}\right) \\
& \mathbf{R}_{\mathbf{z}}\left(\beta_{3}\right) \mathbf{R}_{\mathbf{x}}\left(\gamma_{3}\right) \mathbf{R}_{\mathbf{z}}\left(\delta_{3}\right) \mathbf{R}_{\mathbf{z}}\left(\alpha_{3}\right)=\mathbf{I} .
\end{aligned}
$$

Hence,

$$
\mathbf{R}_{\mathbf{x}}\left(\gamma_{1}\right) \mathbf{R}_{\mathbf{z}}\left(\theta_{1}\right) \mathbf{R}_{\mathbf{x}}\left(\gamma_{2}\right) \mathbf{R}_{\mathbf{z}}\left(\theta_{2}\right) \mathbf{R}_{\mathbf{x}}\left(\gamma_{3}\right) \mathbf{R}_{\mathbf{z}}\left(\theta_{3}\right)=\mathbf{I}
$$

where $\theta_{1}=\delta_{1}+\alpha_{1}+\beta_{2}, \theta_{2}=\delta_{2}+\alpha_{2}+\beta_{3}, \theta_{3}=\delta_{3}+\alpha_{3}+\beta_{1}$.

Observe that equation (2) can be seen as the closure condition of the spherical triangle depicted in Fig. 1 [13]. Then, the analogues of the law of cosines for the angles $\theta_{1}, \theta_{2}$ and $\theta_{3}$ of this spherical triangle allow us to write:

$$
\begin{aligned}
& g_{1}\left(\gamma_{1}, \gamma_{2}, \gamma_{3}, \theta_{1}\right)=\cos \gamma_{1} \cos \gamma_{2}-\cos \gamma_{3}-\sin \gamma_{1} \sin \gamma_{2} \cos \theta_{1}=0, \\
& g_{2}\left(\gamma_{1}, \gamma_{2}, \gamma_{3}, \theta_{2}\right)=\cos \gamma_{3} \cos \gamma_{2}-\cos \gamma_{1}-\sin \gamma_{3} \sin \gamma_{2} \cos \theta_{2}=0, \\
& g_{3}\left(\gamma_{1}, \gamma_{2}, \gamma_{3}, \theta_{3}\right)=\cos \gamma_{3} \cos \gamma_{1}-\cos \gamma_{2}-\sin \gamma_{3} \sin \gamma_{1} \cos \theta_{3}=0 .
\end{aligned}
$$

Finally, 


$$
\begin{aligned}
& \theta_{1}= \pm \arccos \left(\frac{\cos \gamma_{1} \cos \gamma_{2}-\cos \gamma_{3}}{\sin \gamma_{1} \sin \gamma_{2}}\right) \\
& \theta_{2}= \pm \arccos \left(\frac{\cos \gamma_{3} \cos \gamma_{2}-\cos \gamma_{1}}{\sin \gamma_{3} \sin \gamma_{2}}\right) \\
& \theta_{3}= \pm \arccos \left(\frac{\cos \gamma_{3} \cos \gamma_{1}-\cos \gamma_{2}}{\sin \gamma_{3} \sin \gamma_{1}}\right)
\end{aligned}
$$

If we take one solution for any of these three angles, the solution for the other two becomes unique. Thus, we have two sets of solutions. To avoid checking the eight combinations of signs to obtain the two right combinations, we can express, for example, $\theta_{2}$ and $\theta_{3}$ in terms of $\theta_{1}$. To this end, applying the law of sines to the spherical triangle in Fig. 1, we have:

$$
\begin{aligned}
\sin \theta_{2} & =\frac{\sin \gamma_{1}}{\sin \gamma_{3}} \sin \theta_{1}, \\
\sin \theta_{3} & =\frac{\sin \gamma_{2}}{\sin \gamma_{3}} \sin \theta_{1} .
\end{aligned}
$$

Therefore,

$$
\begin{aligned}
& \theta_{2}=\operatorname{atan} 2\left(\frac{\sin \gamma_{1}}{\sin \gamma_{3}} \sin \theta_{1}, \frac{\cos \gamma_{3} \cos \gamma_{2}-\cos \gamma_{1}}{\sin \gamma_{3} \sin \gamma_{2}}\right), \\
& \theta_{3}=\operatorname{atan} 2\left(\frac{\sin \gamma_{2}}{\sin \gamma_{3}} \sin \theta_{1}, \frac{\cos \gamma_{3} \cos \gamma_{1}-\cos \gamma_{2}}{\sin \gamma_{3} \sin \gamma_{1}}\right) .
\end{aligned}
$$

As an example, consider the problem solved in [4] and [7] (note that the latter reference contains several typos in the provided numerical values). In this particular case, $\mathbf{n}_{1}=\left(\cos 80^{\circ} \cos 45^{\circ}, \cos 80^{\circ} \sin 45^{\circ}, \sin 80^{\circ}\right), \mathbf{n}_{2}=\left(\sin 60^{\circ}\right.$, $\left.\cos 60^{\circ}, 0\right), \mathbf{n}_{3}=(1,0,0), \mathbf{n}=\left(\cos 50^{\circ} \cos 25^{\circ}, \cos 50^{\circ} \sin 25^{\circ}, \sin 50^{\circ}\right)$, and $\phi=60^{\circ}$. Using the procedure given above, we obtain

$$
\begin{array}{lll}
\delta_{1}=90^{\circ}, & \beta_{2}=143.03^{\circ}, & \theta_{1}= \pm 93.24^{\circ}, \\
\delta_{2}=-27.39^{\circ}, & \beta_{3}=-60^{\circ}, & \theta_{2}= \pm 91.88^{\circ}, \\
\delta_{3}=132.01^{\circ}, & \beta_{1}=29.78^{\circ}, & \theta_{3}= \pm 149.58^{\circ} .
\end{array}
$$

The resulting two sets of solutions are $\left\{\alpha_{1}=33.73^{\circ}, \alpha_{2}=-4.50^{\circ}, \alpha_{3}=\right.$ $\left.48.63^{\circ}\right\}$ and $\left\{\alpha_{1}=-139.79^{\circ}, \alpha_{2}=179.27^{\circ}, \alpha_{3}=-12.21^{\circ}\right\}$, which coincide with those given in [4] and [7]. 


\section{Applying the principle of transference}

All the presentations of the principle of transference invariably resort to the algebraic structure of dual numbers, but for our purposes it is better to avoid it to derive a more easily implementable formulation. To this end, let us suppose that a loop closure conditions in a spherical mechanism can be expressed as:

$$
g_{1}\left(\xi_{1}, \ldots, \xi_{n}\right)=0, \ldots, g_{m}\left(\xi_{1}, \ldots, \xi_{n}\right)=0
$$

where $\xi_{i}, i=1, \ldots, n$ represent rotations about the axes $\mathbf{n}_{1}, \mathbf{n}_{2}, \ldots, \mathbf{n}_{n}$, respectively. Then, if we do not only rotate but, at the same time, we also translate along the directions defined by these axes the quantities $d_{1}, d_{2}, \ldots, d_{n}$, respectively, these translations cannot be assigned independently, they must satisfy the following $n$ relationships:

$$
\sum_{i=1}^{n} d_{i} \frac{\partial g_{1}}{\partial \xi_{i}}=0, \ldots, \sum_{i=1}^{n} d_{i} \frac{\partial g_{m}}{\partial \xi_{i}}=0
$$

where the sign of $d_{i}$ is imposed by the sign of $\xi_{i}$ according to the right hand rule (see [14] for details).

Now, if we apply the principle of transference, as stated above, to (3), (4), and (5), we obtain:

$$
\left(\begin{array}{lll}
\mathbf{a}_{1} & \mathbf{a}_{2} & \mathbf{a}_{3}
\end{array}\right)\left(\begin{array}{l}
d_{\gamma_{1}} \\
d_{\gamma_{2}} \\
d_{\gamma_{3}}
\end{array}\right)+\left(\begin{array}{lll}
\mathbf{b}_{1} & \mathbf{b}_{2} & \mathbf{d}_{3}
\end{array}\right)\left(\begin{array}{l}
d_{\theta_{1}} \\
d_{\theta_{2}} \\
d_{\theta_{3}}
\end{array}\right)=0
$$

where $d_{\gamma_{1}}, d_{\gamma_{2}}, d_{\gamma_{3}}, d_{\theta_{1}}, d_{\theta_{2}}$, and $d_{\theta_{3}}$ correspond to translations along the axes associated with the rotation angles $\gamma_{1}, \gamma_{2}, \gamma_{3}, \theta_{1}, \theta_{2}$, and $\theta_{3}$, respectively, and

$$
\begin{aligned}
& \mathbf{a}_{1}=\left(\begin{array}{c}
-\sin \gamma_{1} \cos \gamma_{2}-\cos \gamma_{1} \sin \gamma_{2} \cos \theta_{1} \\
\sin \gamma_{1} \\
-\cos \gamma_{3} \sin \gamma_{1}-\sin \gamma_{3} \cos \gamma_{1} \cos \theta_{3}
\end{array}\right), \mathbf{b}_{1}=\left(\begin{array}{c}
\sin \gamma_{1} \sin \gamma_{2} \sin \theta_{1} \\
0 \\
0
\end{array}\right), \\
& \mathbf{a}_{2}=\left(\begin{array}{c}
-\cos \gamma_{1} \sin \gamma_{2}-\sin \gamma_{1} \cos \gamma_{2} \cos \theta_{1} \\
-\cos \gamma_{3} \sin \gamma_{2}-\sin \gamma_{3} \cos \gamma_{2} \cos \theta_{2} \\
\sin \gamma_{2}
\end{array}\right), \mathbf{b}_{2}=\left(\begin{array}{c}
0 \\
\sin \gamma_{3} \sin \gamma_{2} \sin \theta_{2} \\
0
\end{array}\right), \\
& \mathbf{a}_{3}=\left(\begin{array}{c}
\sin \gamma_{3} \\
-\sin \gamma_{3} \cos \gamma_{2}-\cos \gamma_{3} \sin \gamma_{2} \cos \theta_{2} \\
-\sin \gamma_{3} \cos \gamma_{2}-\cos \gamma_{3} \sin \gamma_{1} \cos \theta_{3}
\end{array}\right), \mathbf{b}_{3}=\left(\begin{array}{c}
0 \\
\sin \gamma_{3} \sin \gamma_{1} \sin \theta_{1}
\end{array}\right) .
\end{aligned}
$$

In the next section, these formulas are used to solve the inverse kinematics of a $3 \mathrm{C}$ manipulator. 


\section{Example: Inverse kinematics of the $3 \mathrm{C}$ manipulator}

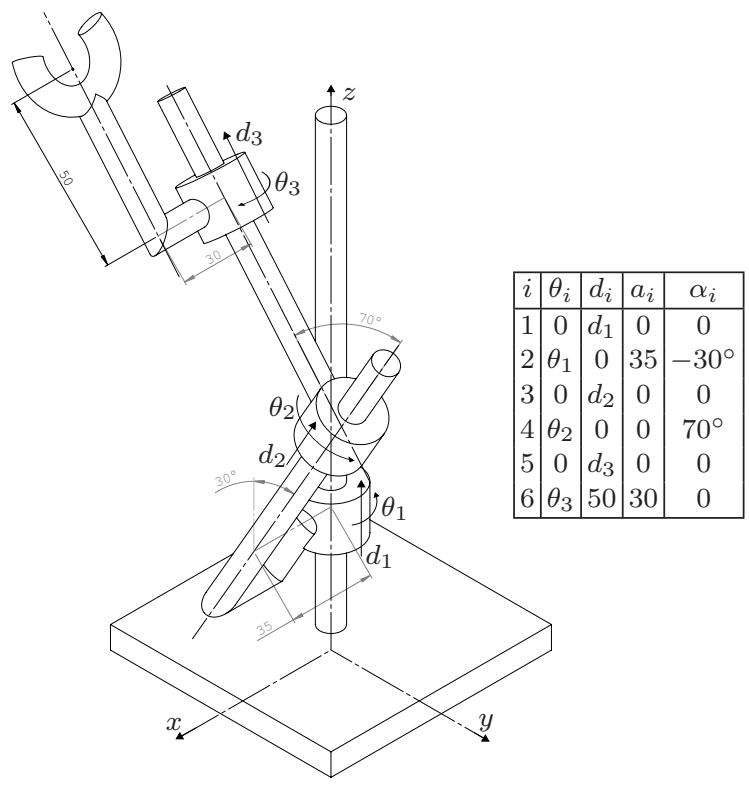

Fig. 2 A 3C manipulator and its DH-parameters.

Clearly, calculating the inverse kinematics of an oblique spherical wrist ${ }^{1}$ is equivalent to solving (1) for $\alpha_{1}, \alpha_{2}$, and $\alpha_{3}$. As a generalization, it can be observed that calculating the inverse kinematics of serial manipulator with three cylindrical joints can be reduced to solve the same set of equations to obtain the revolute joint angles, and the system of equations in (6) to obtain the prismatic joint displacements.

The inverse kinematics of a general 3C manipulator was solved for the first time in [15]. Since then, this has remained the standard reference for this kind of robot. To exemplify the equivalence between calculating the inverse kinematics of 3C manipulators and calculating generalized dual Euler angles, let us considered the $3 \mathrm{C}$ manipulator shown in Fig. 2. Solving its inverse kinematics reduces to solve the matrix equation

$$
\begin{array}{r}
\mathbf{T}_{\mathbf{z}}\left(d_{1}\right) \mathbf{R}_{\mathbf{z}}\left(\theta_{1}\right) \mathbf{R}_{\mathbf{x}}\left(-30^{\circ}\right) \mathbf{T}_{\mathbf{x}}(35) \mathbf{T}_{\mathbf{z}}\left(d_{2}\right) \mathbf{R}_{\mathbf{z}}\left(\theta_{2}\right) \mathbf{R}_{\mathbf{x}}\left(70^{\circ}\right) \\
\mathbf{T}_{\mathbf{z}}\left(d_{3}\right) \mathbf{R}_{\mathbf{z}}\left(\theta_{3}\right) \mathbf{T}_{\mathbf{z}}(50) \mathbf{T}_{\mathbf{x}}(30)=\mathbf{E},
\end{array}
$$

where $\mathbf{E}$ represents the location of its end-effector. If we set

\footnotetext{
${ }^{1}$ A spherical wrist is said to be simple if $\mathbf{n}_{1} \perp \mathbf{n}_{2}$ and $\mathbf{n}_{2} \perp \mathbf{n}_{3}$ or oblique, otherwise.
} 

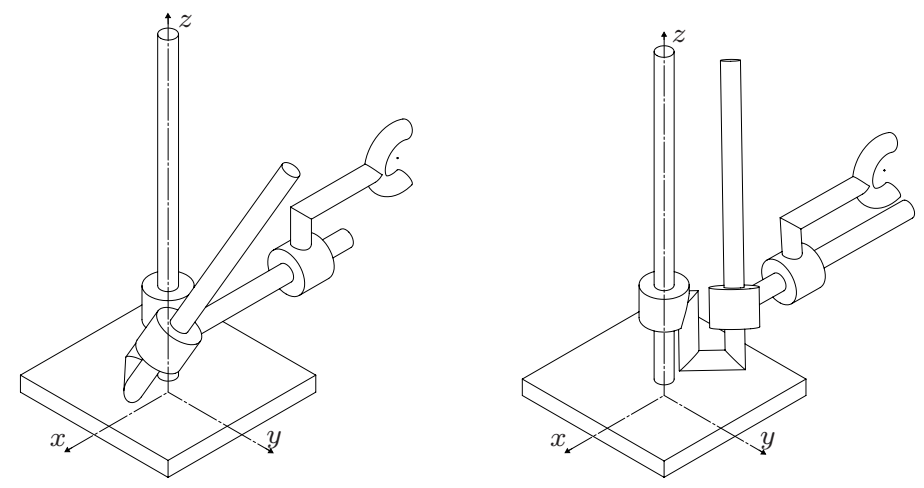

Fig. 3 The two inverse kinematics solutions of the analyzed manipulator for a particular location of its end-effector (see text for details).

$$
\mathbf{E}=\left(\begin{array}{cccc}
0 & 0 & -1 & -115 \\
0 & 1 & 0 & 25 \\
1 & 0 & 0 & 85 \\
0 & 0 & 0 & 1
\end{array}\right)
$$

and calculate the ZXZ dual Euler angles of $\mathbf{E T}_{\mathbf{x}}(-30) \mathbf{T}_{\mathbf{z}}(-50)$, (7) can be rewritten as:

$$
\begin{aligned}
\mathbf{T}_{\mathbf{z}}\left(d_{1}\right) \mathbf{R}_{\mathbf{z}}\left(\theta_{1}\right) \mathbf{R}_{\mathbf{x}}\left(-30^{\circ}\right) \mathbf{T}_{\mathbf{x}}(35) \mathbf{T}_{\mathbf{z}}\left(d_{2}\right) \mathbf{R}_{\mathbf{z}}\left(\theta_{2}\right) \mathbf{R}_{\mathbf{x}}\left(70^{\circ}\right) \mathbf{T}_{\mathbf{z}}\left(d_{3}\right) \mathbf{R}_{\mathbf{z}}\left(\theta_{3}\right) \\
=\mathbf{R}_{\mathbf{z}}\left(-90^{\circ}\right) \mathbf{T}_{\mathbf{z}}(55) \mathbf{R}_{\mathbf{x}}\left(90^{\circ}\right) \mathbf{T}_{\mathbf{x}}(-25) \mathbf{R}_{\mathbf{z}}\left(90^{\circ}\right) \mathbf{T}_{\mathbf{z}}(65)
\end{aligned}
$$

After rearranging terms, we finally obtain:

$$
\begin{array}{r}
\mathbf{R}_{\mathbf{x}}\left(-90^{\circ}\right) \mathbf{T}_{\mathbf{x}}(25) \mathbf{R}_{\mathbf{z}}\left(\theta_{1}+90^{\circ}\right) \mathbf{T}_{\mathbf{z}}\left(d_{1}-55\right) \mathbf{R}_{\mathbf{x}}\left(-30^{\circ}\right) \mathbf{T}_{\mathbf{x}}(35) \\
\mathbf{R}_{\mathbf{z}}\left(\theta_{2}\right) \mathbf{T}_{\mathbf{z}}\left(d_{2}\right) \mathbf{R}_{\mathbf{x}}\left(70^{\circ}\right) \mathbf{R}_{\mathbf{z}}\left(\theta_{3}-90^{\circ}\right) \mathbf{T}_{\mathbf{z}}\left(d_{3}-65\right)=\mathbf{I} .
\end{array}
$$

After dropping translations and proceeding as explained in Section 2, the following two sets of revolute joint angles are obtained: $\left\{\theta_{1}=43.16^{\circ}\right.$, $\left.\theta_{2}=-129.08^{\circ}, \theta_{3}=67.16^{\circ}\right\}$, and $\left\{\theta_{1}=-223.16^{\circ}, \theta_{2}=129.08^{\circ}, \theta_{3}=112.84^{\circ}\right\}$. Then, substituting these results in (6), and solving the resulting linear system in $d_{1}, d_{2}$ and $d_{3}$, we obtain the following sets of translations for the prismatic joints: $\left\{d_{1}=52.49, d_{2}=2.9, d_{3}=89.54\right\}$, and $\left\{d_{1}=57.51, d_{2}=-2.9, d_{3}=40.46\right\}$, respectively. In Fig. 3, the robot is represented in both configurations. 


\section{Conclusions}

We have presented an alternative simple procedure to calculate sets of generalized Euler angles. Next, this procedure has been extended from spherical to spatial kinematics using a variation of the principle of transference that does not rely on the use of dual angles. The arising equations have been shown to be equivalent to those resulting from solving the inverse kinematics of the general 3C robot, a problem already solved using dual orthogonal matrices by Pennock and Vierstra in 1990. Probably due to the fact that, in the example presented by these authors, the axes of the cylindrical joints are orthogonal, their work has been overlooked in recent papers dealing with generalizations of Euler angles.

\section{References}

1. Stuelpnagel, J.: On the parametrization of the three-dimensional rotation group. SIAM Review, 6(4), 422-430 (1964)

2. Davenport, P.B.: Rotations about nonorthogonal axes, AIAA Journal, 11(6), 853-857 (1973)

3. Dimentberg, F.M.: Theory of Screws and its Applications, Nauka, Moscow, (1978)

4. Wohlhart, K.: Decomposition of a finite rotation into three consecutive rotations about given axis. In: Proceedings of the 6th int. Conf. on the Theory of Machines and Mechanisms, Lieberec, Czechoslovakia, 325-332 (1992)

5. Wittenburg, J. and Lilov, L.: Decomposition of a finite rotation into three rotations about given axes, Multibody System Dynamics, 9(4), 353-375 (2003)

6. Shuster, M.D. and Markley, F.L.: Generalization of the Euler angles, The Journal of the Astronautical Sciences, 51(2), 123-132 (2003)

7. Mladenova, C.D. and Mladenov, I.M.: Vector decomposition of finite rotations, Reports on Mathematical Physics, 69(1), 107-117 ( 2011)

8. Piovan, G. and Bullo, F.: On coordinate-free rotation decomposition: Euler angles about arbitrary axes, IEEE Transactions on Robotics, 28(3), 728-733 (2012)

9. Baker, J. E. and Parkin, I. A.: On compounding three successive finite displacement screws, Advances in Robot Kinematics: Analysis and Control, 337-342, Springer (1998)

10. Ying, N. and Kim, W.: Use of dual Euler angles to quantify the three-dimensional joint motion and its application to the ankle joint complex, Journal of Biomechanics, 35(12), 1647-1657 (2002)

11. Teua, K.K., Kimb, W., Fussa, F.K. and Tanc, J.: The analysis of golf swing as a kinematic chain using dual Euler angle algorithm, Journal of Biomechanics, 39(7), 1227-1238 (2006)

12. Rico-Martínez, J.M. and Duffy, J.: The principle of transference: history, statement and proof. Mechanism and Machine Theory, 28(1), 165-177 (1993)

13. Duffy, J. and Rooney, J.: A foundation for a unified theory of analysis of spatial mechanisms, Journal of Engineering for Industry. Transactions of the ASME, 97(4), 1159-1164 (1975)

14. Thomas, F.: On the n-bar mechanism, or how to find global solutions to redundant single loop spatial kinematic chains. In: Proceedings of the 1992 IEEE International Conference on Robotics and Automation, I, 403-408 (1992)

15. Pennock, G.R. and Vierstra, B.C.: The inverse kinematics of a three-cylindric robot, International Journal of Robotics Research, 9(4), 75-85 (1990) 